\title{
Differentiation of the acidic groups of fulvic acids from lignite by potentiometric titration in acetone, acetonitrile and isopropanol
}

\author{
José M. Andrés and Clemente Romero \\ Instituto de Carboquímica, C.S.I.C., Pza. Paraíso no. 1, Apdo. 589, 50004-Zaragoza, Spain \\ (Received 2 November 1987)
}

\begin{abstract}
Potentiometric titrations in acetone, acetonitrile and isopropanol, with tetrabutylammonium hydroxide as titrant, were carried out to differentiate between the acidic groups of fulvic acids. Titrations in pure solvents were able to differentiate at least three acidic groups of different acidic strength. The amount of acidic hydrogen titrated increased with the solvent's basicity: isopropanol < acetone $<$ acetonitrile. The addition of small amounts of water to the solvents had a strong effect on the titrations, leading to very similar values for the three inflection points found in each solvent. This was attributed to preferential solvation of fulvic acids by water
\end{abstract}

(Keywords: lignite; analysis; solvents)

\begin{abstract}
Humic substances are complex acidic materials with carboxyl and hydroxyl (aromatic and aliphatic) groups as their main functional groups. Most of the work on the determination and differentiation of the acidic groups has been carried out by aqueous potentiometric titrations ${ }^{1-6}$ using numerical methods to differentiate up to six groups of different acidic strength. On the other hand, nonaqueous potentiometric titrations have
\end{abstract}

been used to a small extent ${ }^{7-11}$, using dimethylformamide or dimethylsulphoxide as solvents.

A preliminary survey for the application of non-aqueous titrations to humic substances was undertaken and a previous paper $^{11}$ reported the results obtained using dimethylformamide and dimethylsulphoxide as solvents together with potassium and tetrabutylammonium hydroxides (TBAH) as

Table 1 Titration of fulvic acids in various solvents using TBAH as titrant and glass-calomel electrodes

\begin{tabular}{|c|c|c|c|c|c|c|}
\hline Solvent & $\delta \mathrm{E}(\mathrm{mV})$ & End-po & ts (in $\mathrm{m}$ & $g^{-1}$ fulv & ic acids) & \\
\hline Isopropanol & 800 & $\begin{array}{c}0.30 \\
(0.04)\end{array}$ & $\begin{array}{r}3.34^{a} \\
(0.11)\end{array}$ & $\begin{array}{c}5.30 \\
(0.23)\end{array}$ & & \\
\hline Acetone & 1050 & $\begin{array}{r}2.07^{a} \\
(0.21)\end{array}$ & $\begin{array}{r}3.28^{a} \\
(0.32)\end{array}$ & $\begin{array}{r}5.17^{a} \\
(0.02)\end{array}$ & $\begin{array}{l}5.76 \\
(0.16)\end{array}$ & \\
\hline Acetonitrile & 1000 & & $\begin{array}{l}0.32 \\
(0.24)\end{array}$ & $\begin{array}{r}5.05^{a} \\
(0.08)\end{array}$ & & $\begin{array}{c}6.37 \\
(0.04)\end{array}$ \\
\hline Isopropanol +5 vol $\%$ water & 680 & $\begin{array}{c}0.49 \\
(0.05)\end{array}$ & & $\begin{array}{r}5.11^{a} \\
(0.11)\end{array}$ & & $\begin{array}{r}6.00^{a} \\
(0.11)\end{array}$ \\
\hline Acetone $+5 \mathrm{vol} \%$ water & 825 & $\begin{array}{l}0.49 \\
(0.01)\end{array}$ & & $\begin{array}{r}5.11^{a} \\
(0.11)\end{array}$ & & $\begin{array}{c}6.28 \\
(0.12)\end{array}$ \\
\hline Acetonitrile +5 vol $\%$ water & 770 & $(0.48-1.60)^{a}$ & & $\begin{array}{r}4.95^{a} \\
(0.03)\end{array}$ & & $\begin{array}{c}6.34 \\
(0.10)\end{array}$ \\
\hline Average wet solvents & & $\begin{array}{c}0.49 \\
(0.02)\end{array}$ & & $\begin{array}{c}5.03 \\
(0.10)\end{array}$ & & $\begin{array}{c}6.22 \\
(0.19) \\
675 b\end{array}$ \\
\hline
\end{tabular}

${ }^{a}$ Indicates doubtful inflection point corresponding to a small minimum on the derivative curve

${ }^{b}$ Obtained by acid-base titration with $\mathrm{KOH}$

Values in parentheses indicate standard deviation

titrants to differentiate the acidic groups of fulvic acids from a Spanish lignite. This paper reports the results obtained with isopropanol, acetone and acetonitrile, all good differentiating solvents for carboxylic acids ${ }^{1-14}$ and the effect of water on the titration. TBAH was used as titrant because $\mathrm{KOH}$ led to precipitation when used with these solvents.

\section{EXPERIMENTAL}

Fulvic acids preparation, apparatus and end-point determination techniques are as described previously ${ }^{11}$. Glass-calomel electrodes were used. The solvents used (acetone, acetonitrile and isopropanol) were of reagent grade (RG) quality, and were distilled and stored in a nitrogen atmosphere. $\mathrm{CO}_{2}$-free water was obtained by distillation of deionized water under nitrogen. Tetrabutylammonium hydroxide, also $R G$ quality, was dissolved in isopropanol and standardized daily against benzoic acid. The titrant concentrations used were 0.2 and $0.08 \mathrm{~N}$. The concentration of fulvic acids titrated ranged from 0.58 to $1.71 \mathrm{~g}^{-1}$.

\section{RESULTS AND DISCUSSION}

As discussed previously ${ }^{11}, \mathrm{dV} / \mathrm{dE}$ was the most successful method for determining inflection points on the titration curve. 


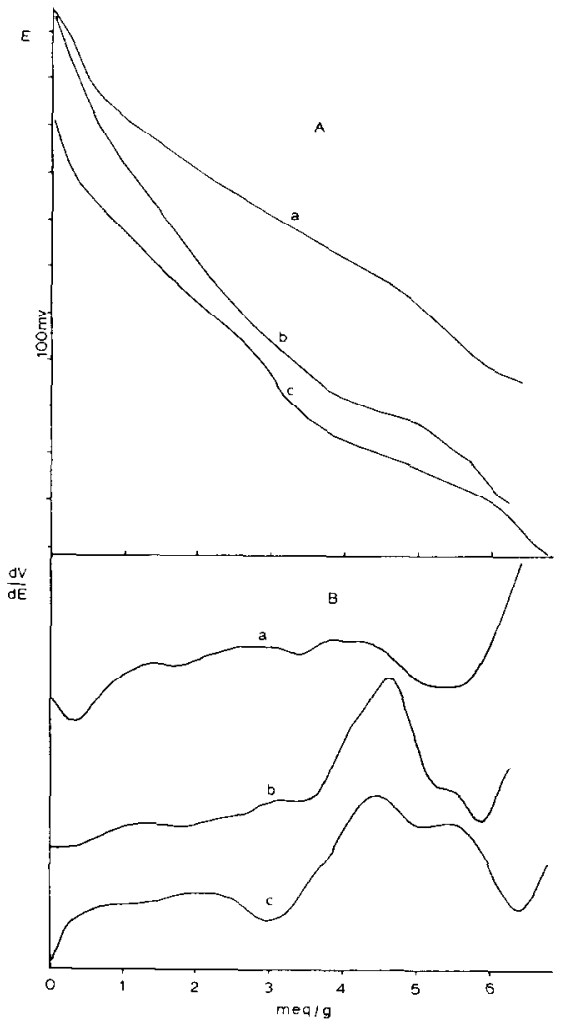

Figure 1 Titration of fulvic acids in: a, isopropanol; b, acetone; $c$, acetonitrile using TBAH as titrant. A, titration curves; B, derivative $(\mathrm{dV} / \mathrm{dE})$ curves

These appear as minima on the derivative curves. The shape of these curves is concentration dependent, but the values calculated remained constant for the concentrations studied as shown in Table 1 , which also includes the precision of the measurements. Additional information about potential ranges and exchangeable acidity is also included.

\section{Titration in anhydrous solvents}

The major drawback to the use of the above-mentioned titration media is the low solubility of fulvic acids in the anhydrous solvents. This led to long times to equilibrium, and this effect was more pronounced when approaching inflection points. No attempts were made to quantitatively determine the solubility parameters but visual inspection showed that the solubility of the fulvic acids increased with the basicity of the solvent.

Figure 1 shows titration curves and derivative plots obtained for the titrations in anhydrous solvents. As reported for DMSO and DMF ${ }^{11}$, TBAH was unable to resolve completely the first part of the titration. In general, intermediate inflection points were small and their calculation is favoured by increasing the concentration of the sample to be titrated. On the other hand, final end-points give sharp minima and are easily calculated.
The total titratable hydrogen increased with the basicity of the solvent, in the following order: $H_{t}$ (isopropanol) $<H_{t}$ (acetone) $<\mathrm{H}_{1}$ (acetonitrile) and no close agreement was obtained between the inflection points in each solvent, except for the small minima appearing at about 3.3 and $5.1 \mathrm{meq}^{-1}$. This effect could be explained either by the different solvation ability of the solvents tested or, more likely, by the degree of polymer coiling allowed by the polarity of the solvent considered.

\section{Wet solvents}

Titrations were carried out in solvents containing $5 \mathrm{vol} \%$ water to increase the solubility of the fulvic acids. It has been reported $^{15}$ that such a small amount of water docs not modify the differentiating properties of the solvents and the solubility of fulvic acids in the above mentioned solvents was enhanced considerably. The titration and derivative curves obtained in wet solvents are shown in Figure 2.

Table 1 shows a close agreement between the values obtained in all wet solvents despite some difficulties in calculating values for the intermediate inflection point (which appears as a shoulder) and for the broad minima appearing at about $5.1 \mathrm{meqg}^{-1}$ in isopropanol and between 0.5 and $1.6 \mathrm{meq} \mathrm{g}^{-1}$ in acetonitrile. The three inflection points obtained seem to be solvent independent, despite the considerable difference shown between them in anhydrous titrations. Consequently average values and standard deviations were calculated for all the results.

There is no ready explanation for this effect, and it is necessary to consider conformational changes during the titration already reported for aqueous titrations ${ }^{2.16}$. Harlow and Wyld ${ }^{15}$ reported the decrease or cancellation of the differentiating properties of organic solvents containing moderate amounts of water, and attributed it to a preferential solvation of ions by water. By a similar argument, the preferential solvation of ionized or ionizable groups hy water could greatly influence the degree of coiling of fulvic acids, equating the properties of the three solvents tested and yielding similar values for the inflection points. This effect of water should depend on its concentration, so cancellation of the differentiation could be expected when using a higher concentration of water in the solvents.

\section{Comparison with other solvents}

Values for this sample of fulvic acids in several solvent-titrant combinations were reported previously ${ }^{11}$. There is no agreement between the values obtained for the inflection points in each solvent The total acidity determined was solvent dependent, as expected, because of the

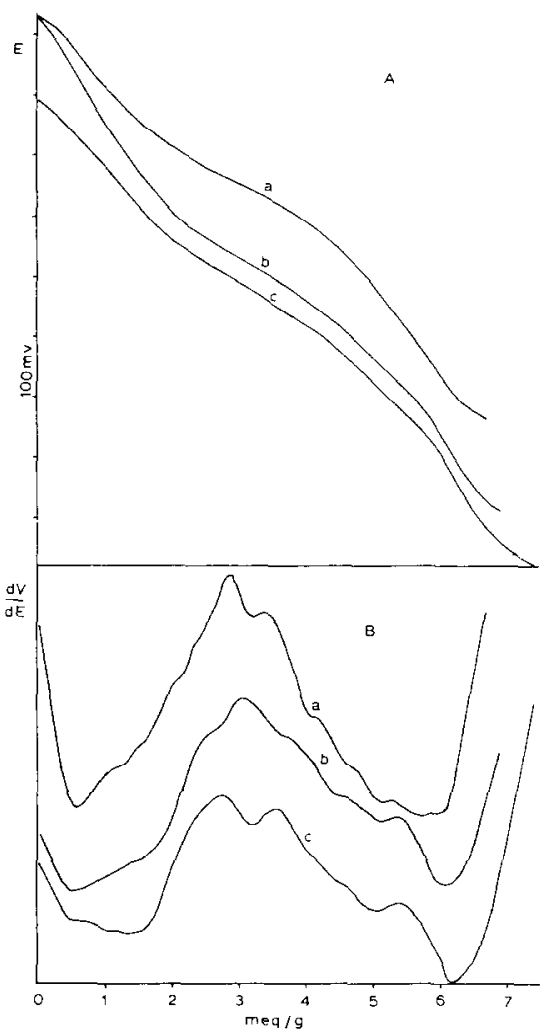

Figure 2 Titration of fulvic acids in: a isopropanol; b, acetone; c, acetonitrile, containing $5 \mathrm{vol} \%$ of water, using TBAH as titrant. A, titration curves; $B,(d V / d E)$ derivative curves

titration of weaker acidic groups in the more basic solvents, but led to a value close to those obtained by aqueous titration in the more basic solvents. On the other hand, the value obtained for the total acidity in isopropanol, in agreement with intermediate inflection points obtained in the other solvents tested in this paper, should reflect the whole concentration of carboxyl groups, so protonated amines or, more likely, activated phenols should account for the rest of the acidic groups titrated in other solvents.

The number of acidic groups differentiated is rather low and the values obtained in the different combinations used are not in agreement. This could be attributed to clustering of carboxyl groups as a function of the solvation ability of the solvents. In view of the complex nature of humic substances, it is possible that a different degree of coiling as a function of the polarity of the solvent used, prevents the access of the reagent to the inner acidic groups and leads to different intermediate inflection points. The cancellation of this effect by the addition of $5 \mathrm{vol} \%$ of water seems to support this hypothesis.

The method used, especially the titration in wet solvents, appears to have some advantages over aqueous titrations, the most important being the ability to 
resolve groups of different acidic strength in a reproducible way, but extension to other kind of humic substances is necessary to assess the usefulness of this method in dealing with more complex humic structures.

\section{REFERENCES}

1 Gamble, D. S. Can. J. Chem. 1970, 48, 2662
2 Sposito, G. and Holtzclaw, K. M. Soil Sci. Soc. Am. J. 1977, 41, 330

3 Sposito, G., Holtzclaw, K. M. and Keech, D. A. Soil Sci. Soc. Am. J. 1977, 41, 1119

4 Davis, H. and Mott, C. J. R. J. Soil Sci. 1981, 32, 379

5 Arp, P. A. Can. J. Chem. 1983, 61, 1671

6 Paxeus, N. and Wedborg, M. Anal. Chim. Acta 1985, 169, 87

7 van Dijk, H. Sci. Proc, Roy. Dublin Soc. A $1960,1,163$

8 Wright, J. R. and Schnitzer, M. Trans. 7th Int Congr. Soil Sci., 1960, 2, 120

9 Thompson, S. O. and Chesters, G. J. Soil Sci. $1969,20,346$
10 Yonebayashi, K, and Hattori, T. Org. Geochem. 1985, 8, 47

11 Andrés, J. M., Romero, C. and Gavilán, J. M. Talanta 1987, 34, 583

12 Harlow, G. A., Noble, C. M. and Wyld, G. E. A. Anal. Chem. 1956, 28, 787

13 Fritz, J. S. and Keen, R. T. Anal. Chem. $1953,25,179$

14 Fritz, J. S. and Yamamura, S. S. Anal. Chem. 1957, 29, 1079

15 Harlow, G. A. and Wylde, G. E. A. Anal. Chem. 1958, 30, 69

16 Varney, M. S., Mantoura, R. F. C. Whitfield, M., Turner, D. R. and Riley, J. P. NATO Conf. Ser., (Ser) 4, 1983, 9, 75

\title{
Chromatographic separation and characterization of asphaltene subfractions from Saudi Arabian crudes
}

\author{
Misbah-ul-Hasan, M. Nahid Siddiqui* and M. Arab* \\ Fuel Research Centre, PCSIR, Karachi-39, Pakistan \\ * Department of Chemistry. University of Petroleum and Minerals, Dhahran, Saudi Arabia \\ (Received 23 November 1987; revised 9 February 1988)
}

\begin{abstract}
Asphaltenes from two commercial Saudi $\Lambda$ rabian crudes have been separated chromatographically into acids, bases and residue fractions, which were analysed by elemental analysis, ${ }^{1} \mathrm{H}$ and ${ }^{13} \mathrm{C} \mathrm{n} . \mathrm{m} . \mathrm{r}$. and i.r. spectroscopy. The acid fraction was obtained in the highest yield from both asphaltenes. The acids and bases from Arab Heavy asphaltenes had higher $\mathrm{H} / \mathrm{C}$ ratios than those from Arab Berri asphaltenes. The highest content of nitrogen was found in the base fraction of Arab Heavy asphaltenes. N.m.r. spectra indicated a higher percentage of aromatic hydrogen atoms in Arab Berri base fractions, compared with those from Arab Heavy asphaltenes. The acid fraction from Arab Heavy asphaltenes contained more saturated carbon atoms and straight chain paraffin residues, the latter are of longer chain length. Various parameters for aromatic carbons $\left(\mathrm{C}_{\mathrm{ar}}, \mathrm{C}_{\mathrm{ar}, \mathrm{alk}}, \mathrm{C}_{\mathrm{ar}, \mathrm{H}}\right.$ and $\left.\mathrm{C}_{\mathrm{ar}, \mathrm{b}}\right)$ wcre calculated for the acid and base fractions of both asphaltenes. The percentages of aromatic carbons, $C_{a r}$, unsubstituted aromatic carbons, $C_{a r . H}$, and bridgehead carbons, $C_{a r, b}$ were all found to be greatest in the acid fraction from Arab Berri asphaltenes.
\end{abstract}

(Keywords: chromatography; crude oil; asphaltene)

Asphaltenes $^{1-3}$ are an important constituent of all crude oils, and may be present to the extent of 5 to $10 \%$. Characterization of asphaltenes can provide useful information about the nature of the crude. Since asphaltenes are themselves very complex, their separation into various fractions assists characterization. Of the different separation schemes ${ }^{4}$, separation into acids, bases and residues is found to be most useful and informative. Structural characterization of these fractions can be carried out by various techniques such as infrared $^{5,6}$ and n.m.r. spectroscopy ${ }^{7-9}$, the latter providing average structural parameters. The procedure for isolation of these asphaltenes from the crude oil has been reported previously ${ }^{10}$. In this paper the separation of asphaltenes, previously isolated ${ }^{10}$ from two commercially available Saudi crudes, into acids, bases and residues and the characterization of these fractions by spectroscopic techniques is presented.

\section{EXPERIMENTAL}

The two Saudi crudes examined, Arab Heavy and Arab Berri, were obtained from the Arabian American Oil Company (ARAMCO). The isolation of asphaltenes from these crudes has been previously described ${ }^{10}$.

\section{Materials}

Anion-exchange 1esin Amberlyst A-27 and cation-exchange resin Amberlyst A15 were obtained from the Rohm and Haas Co. n-Pentane (Fluka AG) was purified by distillation followed by percolating through silica gel $(0.13-$ $0.25 \mu \mathrm{m}$ ) which was previously activated by heating at $250^{\circ} \mathrm{C}$ for about $24 \mathrm{~h}$ and stored in a desiccator. Benzene, methanol and cyclohexane were similarly purified by percolation through silica gel. Isopropylamine (Fluka) was used as received. The separations were made on a water-jacketted liquid chromatographic column $(1.4 \mathrm{~cm}$ i.d. $\times 120 \mathrm{~cm}$ long $)$ incorporating a recycling arrangement 